\title{
A MULTIVARIATE EXTENSION OF EQUILIBRIUM PRICING TRANSFORMS: THE MULTIVARIATE ESSCHER AND WANG TRANSFORMS FOR PRICING FINANCIAL AND INSURANCE RISKS
}

\author{
BY \\ MASAAKI KIJIMA
}

\begin{abstract}
This paper proposes a multivariate extension of the equilibrium pricing transforms for pricing general financial and insurance risks. The multivariate Esscher and Wang transforms are derived from Bühlmann's equilibrium pricing model (1980) under some assumptions on the aggregate risk. It is shown that the Esscher and Wang transforms coincide with each other when the underlying risks are normally distributed.
\end{abstract}

\section{KEYWORDS}

Equilibrium pricing, Wang transform, Esscher transform, State price density, Gaussian copula.

\section{INTRODUCTION}

In the actuarial literature, there have been developed many probability transforms for pricing financial and insurance risks. Such methods include the variance loading, the standard deviation loading, and the exponential principle. Among them, the most popular pricing method is the Esscher transform given by

$$
\pi(Y)=\frac{E\left[Y \mathrm{e}^{-\theta Y}\right]}{E\left[\mathrm{e}^{-\theta Y}\right]}
$$

for a random variable $Y$ representing risk, where $\theta$ is a positive constant ${ }^{1}$ and $E$ is an expectation operator under a probability measure $P$. The Esscher transform is a simple exponential tilting (or exponential change of measure) and has a sound economic interpretation.

1 This paper treats risk as an asset. A liability with loss variable $X$ can be viewed as a negative asset with gain $Y=-X$. See Wang (2002). 
Recently, Wang (2000) proposed a universal pricing method based on the following transformation:

$$
F^{*}(x)=\Phi\left[\Phi^{-1}(F(x))+\lambda\right]
$$

where $\Phi$ denotes the standard normal cumulative distribution function (CDF for short), $F(x)$ is the CDF for the risk $Y$ and $\lambda$ is a positive constant. The transform is now called the Wang transform and produces a risk-adjusted CDF $F^{*}(x)$. The mean value evaluated under $F^{*}(x)$ will define a risk-adjusted "fair value" of risk $Y$ at some future time, which will then be discounted to the current time using the risk-free interest rate.

The Wang transform not only possesses various desirable properties as a pricing method, but also has a clear economic interpretation which can be justified by a sound economic equilibrium argument for risk exchange. For example, the Wang transform (as well as the Esscher transform) is the only distortion function among the family of distortions that can recover CAPM (the capital asset pricing model) for underlying assets and the Black-Scholes formula for options. See Wang (2002) for details.

On the other hand, in the finance literature, the theory of asset pricing has been developed; the theory is well-developed for the so-called complete markets while there are still many blanks for incomplete markets. The insurance market is certainly incomplete; new attempts are required to develop a better pricing method.

The pricing methods developed in the actuarial literature based on probability transformations (or distortions) are often criticized by finance researchers, because they are not linear pricing functionals ${ }^{2}$, yielding arbitrage opportunities. See, e.g., Harrison and Kreps (1979) for details.

In order to develop a linear pricing method while maintaining probability distortions, we need to develop a multivariate version of such existing methods. To see this, suppose that the underlying risks are described by an $n$-dimensional random vector, $\left(X_{1}, X_{2}, \ldots, X_{n}\right)$ say. The probability space is given by $(\Omega, \mathcal{F}, P)$, where $\mathcal{F}=\sigma\left(X_{1}, X_{2}, \ldots, X_{n}\right)$, the smallest $\sigma$-algebra generated from $\left(X_{1}, X_{2}, \ldots\right.$, $\left.X_{n}\right)$. According to the first fundamental theorem of asset pricing, there is no arbitrage opportunity if and only if there exists a state price density ${ }^{3}$. Recall that the state price density is an $\mathcal{F}$-measurable random variable. Hence, supposing that a state price density is given, $\eta=\eta\left(X_{1}, X_{2}, \ldots, X_{n}\right)$ say, the pricing functional is defined as

$$
\pi(Y)=E[\eta Y]=\int_{\Omega} \eta(\omega) Y(\omega) \mathrm{d} P(\omega)
$$

2 The pricing functional $\pi$ is said to be linear if $\pi(a X+b Y)=a \pi(X)+b \pi(Y)$ for all risks $X, Y$ and constants $a, b$.

3 The second fundamental theorem of asset pricing states that the state price density is unique if and only if the market is complete. See, e.g., Pliska (1997) for details. 
for risk $Y$ that is an $\mathcal{F}$-measurable random variable. The pricing functional $\pi$ is linear due to the linearity of the expectation operator.

The classic Esscher transform (1.1) is a special case of (1.3) where $\eta=\mathrm{e}^{-\theta Y}$ / $E\left[\mathrm{e}^{-\theta Y}\right]$. The Wang transform (1.2) can be derived from (1.3) under a set of assumptions on the aggregate risk. See Wang (2003) for details. The aim of this paper is to find an appropriate state price density that covers all the risks under consideration, extending the Esscher and Wang transforms to the multivariate setting ${ }^{4}$. These extensions are derived from Bühlmann's equilibrium pricing model (1980).

The present paper is organized as follows. In the next section, we review Bühlmann's equilibrium pricing model and derive a multivariate extension of the Esscher transform. Of particular interest is the case of normally distributed risks. It is shown that the multivariate Esscher transform is consistent with CAPM and distorts a normal distribution to another normal distribution with mean vector adjusted by $\operatorname{Cov}(Y, \lambda Z)$, the covariance of risk $Y$ and $\lambda Z$, where $Z$ stands for the aggregate risk, which corresponds to the risk from the market portfolio in CAPM, and $\lambda$ a risk aversion index. Section 3 develops a multivariate version of the Wang transform. It is shown that, when the underlying risks are normally distributed, the multivariate Wang transform coincides with the Esscher counterpart.

\section{BÜHLMANN'S EQUILIBRIUM PRICING MODEL}

Consider risk exchanges among a set of agents $j=1,2, \ldots, n$. Each agent is characterized by his/her utility function $u_{j}(x)$, where $u_{j}^{\prime}(x)>0$ and $u_{j}^{\prime \prime}(x) \leq 0$, and initial wealth $w_{j}$. Suppose that agent $j$ faces a risk of potential loss $X_{j}$ and is willing to buy/sell a risk exchange $Y_{j}$. If agent $j$ is an insurance company, the risk exchange $Y_{j}$ is thought of the sum of all insurance policies sold by $j$. While the original risk $X_{j}$ belongs to agent $j$, the risk exchange $Y_{j}$ can be freely bought/ sold by the agents in the market.

In this pure risk exchange model, Bühlmann (1980) derived the following equilibrium pricing formula for risk $Y$, which is an $\mathcal{F}$-measurable random variable, by assuming that each agent $j$ has an exponential utility function $u_{j}(x)=$ $-\mathrm{e}^{-\lambda_{j} x}, j=1,2, \ldots, n^{5}$ :

$$
\pi(Y)=E[\eta Y], \quad \eta=\frac{\mathrm{e}^{-\lambda Z}}{E\left[\mathrm{e}^{-\lambda Z}\right]}
$$

\footnotetext{
4 A similar attempt has been made by Wang (2005) to extend the Wang transform to a multivariate setting, where the Radon-Nikodym derivative is given by the normalized exponential tilting with special structures such as joint and separate distortions. In contrast, this paper assumes no such structures and derives the multivariate extensions directly from Bühlmann's pricing formula.

5 Because this paper considers the pricing of risky assets, the sign of $\lambda$ in the state price density $\eta$ is opposite to that in Bühlmann (1980).
} 
cf. (1.3), where $Z=\sum_{j=1}^{n} X_{j}$ is the aggregate risk and $\lambda$ is given by

$$
\lambda^{-1}=\sum_{j=1}^{n} \lambda_{j}^{-1}, \quad \lambda_{j}>0
$$

The parameter $\lambda$ is thought of the risk aversion index of the representative agent in the market.

In what follows, we assume that the underlying risks are described by an $n$-dimensional random vector $\boldsymbol{X}=\left(X_{1}, X_{2}, \ldots, X_{n}\right)$ and the $\sigma$-algebra is given by $\mathcal{F}=\sigma\left(X_{1}, X_{2}, \ldots, X_{n}\right)$. Note that a particular risk, $Y$ say, is an $\mathcal{F}$-measurable random variable. That is, there exists an $n$-variate function $h(\boldsymbol{x}), \boldsymbol{x}=\left(x_{1}, x_{2}, \ldots, x_{n}\right)$, such that $Y=h(\boldsymbol{X})$ in general. Particular cases include a singleton, i.e. $Y=X_{j}$ for some $j$, and a portfolio of $\left(X_{1}, X_{2}, \ldots, X_{n}\right)$, i.e. $Y=\sum_{j=1}^{n} w_{j} X_{j}$ for some weights $w_{j}$. Bühlmann's equilibrium pricing formula is then given by

$$
\pi(Y)=\frac{E\left[h(\boldsymbol{X}) \mathrm{e}^{-\lambda Z}\right]}{E\left[\mathrm{e}^{-\lambda Z}\right]}, \quad Z=\sum_{j=1}^{n} X_{j}, \quad Y=h(\boldsymbol{X}) .
$$

The pricing functional $\pi(Y)$ is linear since

$$
\pi(a Y+b V)=\frac{E\left[\{a h(\boldsymbol{X})+b g(\boldsymbol{X})\} \mathrm{e}^{-\lambda Z}\right]}{E\left[\mathrm{e}^{-\lambda Z}\right]}=a \pi(Y)+b \pi(V),
$$

where $V=g(\boldsymbol{X})$ for some $g(\boldsymbol{x})$.

Remark 2.1. Consider a particular risk $Y$, which is so small that $Y$ and $Z-Y$ are approximately independent. In this case, we have from (2.1) that

$$
\pi_{\lambda}(Y)=\frac{E\left[Y \mathrm{e}^{-\lambda Y} \mathrm{e}^{-\lambda(Z-Y)}\right]}{E\left[\mathrm{e}^{-\lambda Y} \mathrm{e}^{-\lambda(Z-Y)}\right]} \approx \frac{E\left[Y \mathrm{e}^{-\lambda Y}\right]}{E\left[\mathrm{e}^{-\lambda Y}\right]},
$$

which is the Esscher transform (1.1). Note however that the resulting functional $\pi_{\lambda}(Y)$ does not satisfy the linearity, since

$$
\frac{E\left[(Y+V) \mathrm{e}^{-\lambda(Y+V)}\right]}{E\left[\mathrm{e}^{-\lambda(Y+V)}\right]} \neq \frac{E\left[Y \mathrm{e}^{-\lambda Y}\right]}{E\left[\mathrm{e}^{-\lambda Y}\right]}+\frac{E\left[V \mathrm{e}^{-\lambda V}\right]}{E\left[\mathrm{e}^{-\lambda V}\right]},
$$

unless $Y$ and $V$ are independent of each other. Also, $\pi_{\lambda}(a Y)=a \pi_{a \lambda}(Y) \neq a \pi_{\lambda}(Y)$.

For the $n$-dimensional random vector $\boldsymbol{X}=\left(X_{1}, X_{2}, \ldots, X_{n}\right)$, suppose that the moment generating function (MGF for short) exists, i.e.

$$
m_{X}(\boldsymbol{t})=E\left[\exp \left\{\sum_{j=1}^{n} t_{j} X_{j}\right\}\right]<\infty, \quad \boldsymbol{t}=\left(t_{1}, t_{2}, \ldots, t_{n}\right),
$$


for all $\boldsymbol{t}$ at a neighborhood of the origin. If the MGF exists, the interchange between the partial derivative and the expectation is permissible. Hence, from (2.1), we have

$$
\pi\left(X_{j}\right)=\frac{\partial_{j} m_{X}(-\lambda \mathbf{1})}{m_{X}(-\lambda \mathbf{1})}=\partial_{j} \log m_{X}(-\lambda \mathbf{1})
$$

where 1 denotes the vector with all components being unity, and where $\partial_{j}$ designates the partial derivative with respect to the $j$ th variable. Hence, if the risky claim $Y$ is a portfolio of $\boldsymbol{X}$, i.e. $Y=\sum_{j=1}^{n} w_{j} X_{j}$, the equilibrium price of $Y$ is given by

$$
\pi(Y)=\sum_{j=1}^{n} w_{j} \partial_{j} \log m_{X}(\lambda \mathbf{1})
$$

This means that, in this context, pricing of financial and insurance risks is equivalent to determining the MGF of the underlying multivariate risks.

Remark 2.2. The pricing formula (2.1) rules out log-normal distributions, because the MGF does not exist for any log-normally distributed random variable. On the other hand, according to Iwaki, Kijima and Morimoto (2001), when each agent has a power utility function $u_{j}(x)=\gamma_{j}^{1-\alpha} x^{\alpha} / \alpha, \gamma_{j}>0$, in Bühlmann's equilibrium setting for the same parameter $\alpha<1$, the state price density is given by $\eta=Z^{-(1-\alpha)} / E\left[Z^{-(1-\alpha)}\right]$. The resulting pricing formula is valid for log-normal distributions.

The pricing formula (2.1) can be described in terms of the change of measures. Suppose for the sake of simplicity that the probability density function (PDF for short) of $\boldsymbol{X}$ exists, which is denoted by $f(\boldsymbol{x})$, under the original measure $P$. Let us define

$$
f *(\boldsymbol{x})=\frac{\mathrm{e}^{-\lambda z}}{E\left[\mathrm{e}^{-\lambda Z}\right]} f(\boldsymbol{x}), \quad z=\sum_{j=1}^{n} x_{j} .
$$

Since $f^{*}(\boldsymbol{x})$ is nonnegative and satisfies

$$
\int_{R^{n}} f^{*}(\boldsymbol{x}) \mathrm{d} \boldsymbol{x}=\frac{E\left[\mathrm{e}^{-\lambda Z}\right]}{E\left[\mathrm{e}^{-\lambda Z}\right]}=1,
$$

the function $f^{*}(\boldsymbol{x})$ can be thought of as a PDF. Denoting the new probability measure under which the PDF for $\boldsymbol{X}$ is $f^{*}(\boldsymbol{x})$ by $P^{*}$, the pricing formula (2.1) can be written as

$$
\pi(Y)=\int_{R^{n}} \frac{h(\boldsymbol{x}) \mathrm{e}^{-\lambda z}}{E\left[\mathrm{e}^{-\lambda Z}\right]} f(\boldsymbol{x}) \mathrm{d} \boldsymbol{x}=\int_{R^{n}} h(\boldsymbol{x}) f^{*}(\boldsymbol{x}) \mathrm{d} \boldsymbol{x} .
$$


Therefore, we obtain Bühlmann's equilibrium price as

$$
\pi(Y)=E^{*}[h(\boldsymbol{X})], \quad Y=h(\boldsymbol{X}),
$$

where $E^{*}$ denotes the expectation operator associated with the new probability measure $P^{*}$; cf. Bühlmann (1980). We shall call the transformation (2.4) the multivariate Esscher transform by an obvious reason ${ }^{6}$.

Remark 2.3. In the case of the univariate Esscher transform, i.e.

$$
f^{*}(x)=\frac{\mathrm{e}^{-\lambda x}}{E\left[\mathrm{e}^{-\lambda X}\right]} f(x), \quad \lambda>0,
$$

the ratio $f^{*}(x) / f(x)$ is strictly decreasing in $x$. Hence, denoting the random variable that follows the new $\operatorname{PDF} f^{*}(x)$ by $X^{*}$, we conclude that $X^{*}$ is smaller than $X$ in the sense of likelihood-ratio ordering. This ordering is stronger than the first order stochastic dominance. See Kijima and Ohnishi (1996) for details. On the other hand, from (1.2), we have $\Phi^{-1}\left(F^{*}(x)\right)>\Phi^{-1}(F(x))$ so that $F^{*}(x)>F(x)$ for all $x$. Therefore, in the case of the univariate Wang transform, we conclude that $X^{*}$ is smaller than $X$ in the sense of first order stochastic dominance. In either case, we have $E^{*}[X]<E[X]$, suggesting a proper (i.e. positive) risk premium.

Of interest is then what will be the new PDF (2.4) under the multivariate Esscher transform. Fortunately, we can determine the transformation explicitly for the normal case, as we shall discuss below.

\subsection{The case of normal distributions}

When $\boldsymbol{X}$ follows an $n$-variate normal distribution, the pricing formula (2.3) becomes extremely simple. Suppose that $\boldsymbol{X}$ has mean vector $\boldsymbol{\mu}=\left(\mu_{1}, \mu_{2}, \ldots, \mu_{n}\right)$ and covariance matrix $\boldsymbol{\Sigma}=\left(\sigma_{i j}\right)$. Then, its MGF is given by ${ }^{7}$

$$
m_{X}(\boldsymbol{t})=\exp \left\{\boldsymbol{\mu}^{\top} \boldsymbol{t}+\frac{\boldsymbol{t}^{\top} \boldsymbol{\Sigma} \boldsymbol{t}}{2}\right\},
$$

where $\mathrm{T}$ denotes the transpose. It follows that

$$
\partial_{j} \log m_{X}(-\lambda \mathbf{1})=\mu_{j}-\lambda \sum_{i=1}^{n} \sigma_{i j} .
$$

6 In Jensen (1995), the measure defined by

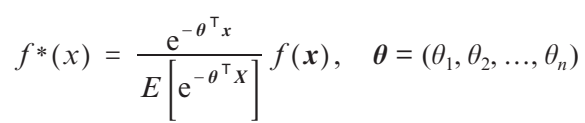

is called an exponentially tilted measure, where T denotes the transpose. The Esscher transform (2.4) is a special case of the exponential tilting with $\boldsymbol{\theta}=\lambda \mathbf{1}$.

7 See, e.g., Tong (1990) for details of multivariate normal distributions. 
Therefore, in the normal case, the multivariate Esscher transform (or Bühlmann's equilibrium price) for risk $Y=\sum_{j=1}^{n} w_{j} X_{j}$ is given by

$$
\pi(Y)=E[Y]-\lambda \operatorname{Cov}(Y, Z),
$$

where

$$
\kappa \equiv \lambda \operatorname{Cov}(Y, Z)=\lambda \sum_{i=1}^{n} \sum_{j=1}^{n} w_{i} \sigma_{i j}
$$

is called the excess loading. Of course, when $Z$ corresponds to the risk from the market portfolio in CAPM, we have

$$
\pi(Z)=E[Z]-\lambda V[Z],
$$

which is the variance loading. It follows from (2.7) and (2.8) that

$$
\frac{E[Y]-\pi(Y)}{E[Z]-\pi(Z)}=\frac{\operatorname{Cov}(Y, Z)}{V[Z]}
$$

where the right hand side is the beta of $Y$ in the theory of CAPM. In other words, the risk premium for $Y$, i.e. $E[Y]-\pi(Y)$, is proportional to the market risk premium, i.e. $E[Z]-\pi(Z)$, and the coefficient is given by the beta for $Y$. Summarizing, we have the following.

Theorem 2.1. Suppose that the underlying risks $\left(X_{1}, X_{2}, \ldots, X_{n}\right)$ follow an n-variate normal distribution. If risk $Y$ is a linear combination of the underlying risks, then the multivariate Esscher transform determines the price $\pi(Y)$ so that

$$
\pi(Y)=E[Y]-\beta_{Y}(E[Z]-\pi(Z)) ; \quad \beta_{Y}=\frac{\operatorname{Cov}(Y, Z)}{V[Z]},
$$

where $\beta_{Y}$ denotes the beta for $Y$ in $C A P M$.

Remark 2.4. Note that the pricing formula (2.9) does not depend on the risk aversion index $\lambda$ of the representative agent. Also, since the beta $\beta_{Y}$ is linear by the definition, the formula (2.9) provides a linear pricing functional. That is,

$$
\begin{aligned}
\pi(a Y+b V) & =E[a Y+b V]-\beta_{a Y+b V}(E[Z]-\pi(Z)) \\
& =a E[Y]+b E[V]-\left(a \beta_{Y}+b \beta_{V}\right)(E[Z]-\pi(Z)) \\
& =a \pi(Y)+b \pi(V),
\end{aligned}
$$

because $\operatorname{Cov}(a Y+b V, Z)=a \operatorname{Cov}(Y, Z)+b \operatorname{Cov}(V, Z)$.

Now, we consider the new $\operatorname{PDF} f^{*}(\boldsymbol{x})$ defined in (2.4) for the normal case. Recall that the $\operatorname{PDF} f(x)$ under $P$ is given by

$$
f(\boldsymbol{x})=K \mathrm{e}^{-Q / 2} ; \quad Q=(\boldsymbol{x}-\boldsymbol{\mu})^{\top} \boldsymbol{\Sigma}^{-1}(\boldsymbol{x}-\boldsymbol{\mu}),
$$


for some normalizing constant $K$, which is determined such that $\int_{R^{n}} f(\boldsymbol{x}) \mathrm{d} \boldsymbol{x}=1$. Note that

$$
(\boldsymbol{x}-\boldsymbol{\mu}-\boldsymbol{\Sigma} \boldsymbol{t})^{\top} \boldsymbol{\Sigma}^{-1}(\boldsymbol{x}-\boldsymbol{\mu}-\boldsymbol{\Sigma} \boldsymbol{t})=Q-2(\boldsymbol{x}-\boldsymbol{\mu})^{\top} \boldsymbol{t}+\boldsymbol{t}^{\top} \boldsymbol{\Sigma} \boldsymbol{t} .
$$

It follows from (2.6) that

$$
\exp \left\{-\frac{1}{2} Q+\boldsymbol{x}^{\top} \boldsymbol{t}\right\}=m_{X}(\boldsymbol{t}) \exp \left\{-\frac{1}{2}(\boldsymbol{x}-\boldsymbol{\mu}-\boldsymbol{\Sigma} \boldsymbol{t})^{\top} \boldsymbol{\Sigma}^{-1}(\boldsymbol{x}-\boldsymbol{\mu}-\boldsymbol{\Sigma} \boldsymbol{t})\right\} .
$$

In particular, when $\boldsymbol{t}=-\lambda \mathbf{1}$, we conclude that

$$
f^{*}(\boldsymbol{x})=K \mathrm{e}^{-\mathrm{Q}^{*} / 2} ; Q^{*}=(\boldsymbol{x}-\boldsymbol{\mu}+\lambda \boldsymbol{\Sigma} \mathbf{1})^{\top} \boldsymbol{\Sigma}^{-1}(\boldsymbol{x}-\boldsymbol{\mu}+\lambda \boldsymbol{\Sigma} \mathbf{1}),
$$

which is the PDF of the $n$-variate normal distribution with mean vector $\boldsymbol{\mu}-\lambda \boldsymbol{\Sigma} \mathbf{1}$ and the same covariance matrix $\boldsymbol{\Sigma}$. The next theorem summarizes ${ }^{8}$.

Theorem 2.2. Suppose that the underlying risks $\boldsymbol{X}=\left(X_{1}, X_{2}, \ldots, X_{n}\right)$ follow an $n$ variate normal distribution with mean vector $\boldsymbol{\mu}$ and covariance matrix $\boldsymbol{\Sigma}$. Then, the multivariate Esscher transform distorts the normal distribution to another normal distribution with mean vector $\boldsymbol{\mu}-\lambda \boldsymbol{\Sigma} \mathbf{1}$ and the same covariance matrix $\boldsymbol{\Sigma}$.

Theorem 2.2 states that, when evaluating a derivative security, $Y=h(\boldsymbol{X})$ say, each risk $X_{j}$ should be adjusted by $\lambda \operatorname{Cov}\left(X_{j}, Z\right)$ for its mean $\mu_{j}$. Hence, when the underlying risks are normal, the price of the derivative is given by

$$
\pi(Y)=E[h(\boldsymbol{X}-\lambda \operatorname{Cov}(\boldsymbol{X}, \boldsymbol{Z}))],
$$

where $\boldsymbol{X}-\lambda \operatorname{Cov}(\boldsymbol{X}, Z)$ denotes the vector whose components are given by $X_{j}-$ $\lambda \operatorname{Cov}\left(X_{j}, Z\right)$. This extends a well-known result in the bivariate case to the multivariate setting .

In particular, when the underlying risks $\left(X_{1}, X_{2}, \ldots, X_{n}\right)$ follow a standard $n$-variate normal distribution with correlation matrix $\boldsymbol{\Sigma}_{\rho}=\left(\rho_{i j}\right)$, the multivariate Esscher transform distorts the standard normal distribution to another normal distribution with mean vector $-\lambda \Sigma_{\rho} \mathbf{1}$ and the correlation matrix $\boldsymbol{\Sigma}_{\rho}$. In this context, suppose that an asset price in the next period under $P$ is given by

$$
S_{i}=K_{i} \exp \left\{\left(\mu_{i}-\frac{\sigma_{i}^{2}}{2}\right)+\sigma_{i} X_{i}\right\}, \quad i=1,2, \ldots, n,
$$

8 Alternatively, the theorem can be proved using the MGF given by (2.6).

9 For any bivariate normal random vector $(X, Y)$, we have

$$
E\left[h(X) \mathrm{e}^{-Y}\right]=E\left[\mathrm{e}^{-Y}\right] E[h(X-\operatorname{Cov}[X, Y])] .
$$

See, e.g. Kijima and Muromachi (2001) for more general results. 
where $\sigma_{i}$ denotes the volatility of asset $i$. According to Theorem 2.2, the current price of asset $i$ is given by

$$
\begin{aligned}
s_{i}(0) & =E^{*}\left[K_{i} \exp \left\{\left(\mu_{i}-\frac{\sigma_{i}^{2}}{2}\right)+\sigma_{i}\left(X_{i}-\lambda \boldsymbol{\Sigma}_{\rho} \mathbf{1}\right)\right\}\right] \\
& =K_{i} \exp \left\{\mu_{i}-\lambda \sigma_{i} \operatorname{Cov}\left(X_{i}, Z\right)\right\},
\end{aligned}
$$

where $Z=\sum_{i=1}^{n} X_{i}$. It follows that

$$
S_{i}=s_{i}(0) \exp \left\{\left(\lambda \sigma_{i} \operatorname{Cov}\left(X_{i}, Z\right)-\frac{\sigma_{i}^{2}}{2}\right)+\sigma_{i} X_{i}\right\}, \quad i=1,2, \ldots, n,
$$

under the original probability measure $P$. Hence, the mean rate of return for asset $i$ must be given by

$$
\mu_{i}=\lambda \sigma_{i} \operatorname{Cov}\left(X_{i}, Z\right), \quad i=1,2, \ldots, n,
$$

in this equilibrium pricing context. This means that the market price of risk for asset $i$ (or risk $X_{i}$ ) is given by $\lambda \operatorname{Cov}\left(X_{i}, Z\right.$ ), not just $\lambda$.

Note that the asset price under the new probability measure $P^{*}$ is given by

$$
S_{i}=s_{i}(0) \exp \left\{-\frac{\sigma_{i}^{2}}{2}+\sigma_{i} X_{i}\right\}, \quad i=1,2, \ldots, n .
$$

Hence, as in Gerber and Shiu (1994), the Black-Scholes formula (1973) is obtained from (2.13) as the current price of a call option written on the asset $S_{i}$. More precisely, the pricing formula

$$
\pi(Y)=\mathrm{e}^{-r} E^{*}\left[\max \left\{S_{i}-K, 0\right\}\right]
$$

provides the well-known Black-Scholes formula, where $S_{i}$ is given by (2.13) under the risk-neutral measure $P^{*}$ and $r$ denotes the risk-free interest rate over the period.

Remark 2.5. Even when $Y$ is a portfolio of $\boldsymbol{X}$, we have

$$
\pi(Y)=E[h(Y-\lambda \operatorname{Cov}(Y, Z))], \quad Y=\sum_{j=1}^{n} w_{j} X_{j} .
$$

Hence, there is no such simple formula as (2.7) for the case of general payoff function $h(x)$. When $\operatorname{Cov}(Y, Z)$ is small, however, we have from the first-order approximation to (2.11) that

$$
\pi(Y) \approx E[h(Y)]-\lambda \operatorname{Cov}(Y, Z) E\left[h^{\prime}(Y)\right] .
$$


Hence, the beta in Theorem 2.1 needs to be modified by the factor $E\left[h^{\prime}(Y)\right]$ for the non-linear payoff function $h(x)$ when the first-order approximation is applied.

\subsection{Archimedean copulas}

In general, the dependence structure of $n$-dimensional random vector $\boldsymbol{X}=\left(X_{1}\right.$, $\left.X_{2}, \ldots, X_{n}\right)$ can be specified by copula. That is, suppose that each marginal CDF of $X_{j}$ is given by $F_{j}(x)$. Then, $F_{j}\left(X_{j}\right)$ follows a standard uniform distribution on $[0,1]$. The copula specifies the joint CDF of $\left(F_{1}\left(X_{1}\right), F_{2}\left(X_{2}\right), \ldots, F_{n}\left(X_{n}\right)\right)$. Denoting the copula by $c(\boldsymbol{x}), \boldsymbol{x}=\left(x_{1}, x_{2}, \ldots, x_{n}\right)$, we have

$$
\begin{aligned}
& P\left\{X_{1} \leq x_{1}, X_{2} \leq x_{2}, \ldots, X_{n} \leq x_{n}\right\} \\
= & P\left\{F_{1}\left(X_{1}\right) \leq F_{1}\left(x_{1}\right), F_{2}\left(X_{2}\right) \leq F_{2}\left(x_{2}\right), \ldots, F_{n}\left(X_{n}\right) \leq F_{n}\left(x_{n}\right)\right\} \\
= & c\left(F_{1}\left(x_{1}\right), F_{2}\left(x_{2}\right), \ldots, F_{n}\left(x_{n}\right)\right) .
\end{aligned}
$$

Hence, we can recover the joint CDF of $\boldsymbol{X}$ from the copula $c(\boldsymbol{x})$. See, e.g., Nelson (1999) for details of copula functions.

The ordinary application of copula to multivariate distributions is to assume a particular copula in an ad hoc manner. Note however that copulas with more than two variables are limited, to the author's best knowledge, to Gaussian copulas, $t$ copulas and Archimedean copulas. While this section considers an Archimedean copula, we employ a Gaussian copula to derive a multivariate Wang transform in the next section ${ }^{10}$.

For any positive random variable $R$, suppose that it has the MGF at a neighbourhood of the origin. Let $\psi(s)=E\left[\mathrm{e}^{-s R}\right], s \geq 0$. It is readily seen that

$$
(-1)^{n} \psi^{(n)}(s)>0, \quad s>0,
$$

where $\psi^{(n)}(s)$ denotes the $n$th derivative of $\psi(s)$. Such a function is called completely monotone. We denote the inverse by $\psi^{-1}(s)$. Suppose further that $\psi(0)=1$ and $\psi(\infty)=0$. Then, the inverse function is decreasing in $s$ with $\psi^{-1}(0)=\infty$ and $\psi^{-1}(1)=0$. Now, for any $\left(x_{1}, \ldots, x_{n}\right) \in(0,1]^{n}$, let

$$
c\left(x_{1}, \ldots, x_{n}\right)=\psi\left(\psi^{-1}\left(x_{1}\right)+\cdots+\psi^{-1}\left(x_{n}\right)\right), \quad 0<x_{j}<1 .
$$

It is well known that the function $c\left(x_{1}, \ldots, x_{n}\right)$ defines an Archimedean copula.

The Archimedean copula can be generated as follows. Consider a family of independent, exponentially distributed random variables with unit mean, $Y_{1}$, $Y_{2}, \ldots, Y_{n}$ say. It is also assumed that $R$ and $Y_{j}$ are independent. Define

$$
X_{j}=F_{j}^{-1}\left[\psi\left(Y_{j} / R\right)\right], \quad j=1,2, \ldots, n,
$$

\footnotetext{
${ }^{10}$ Since there are no MGF's for $t$-distributions, the multivariate Esscher transform does not apply for $t$ copulas in this framework.
} 
where we assume that each CDF $F_{j}(x)$ is strictly increasing for the sake of simplicity. According to Kijima (2002), the $n$-dimensional random vector $\boldsymbol{X}=\left(X_{1}\right.$, $X_{2}, \ldots, X_{n}$ ) has the Archimedean copula (2.14). Hence, given the Archimedean copula, we can generate the random samples $\boldsymbol{x}^{k}=\left(x_{1}^{k}, x_{2}^{k}, \ldots, x_{n}^{k}\right)$ from $\boldsymbol{X}=\left(X_{1}\right.$, $X_{2}, \ldots, X_{n}$ ), and evaluate the expectation (2.1) by Monte Carlo simulation as follows.

\section{Algorithm:}

1. Generate independently random numbers $r^{k}$ and $y_{j}^{k}, j=1,2, \ldots, n$.

2. Set $x_{j}^{k}=F_{j}^{-1}\left[\psi\left(y_{j}^{k} / r^{k}\right)\right], j=1,2, \ldots, n$.

3. Repeat this procedure for sufficiently large $K, k=1,2, \ldots, K$, and evaluate (2.1) approximately as

$$
\pi(Y) \approx \frac{\sum_{k=1}^{K} h\left(\boldsymbol{x}^{k}\right) \mathrm{e}^{-\lambda z^{k}}}{\sum_{k=1}^{K} \mathrm{e}^{-\lambda z^{k}}} ; \quad z^{k}=\sum_{j=1}^{n} x_{j}^{k} .
$$

Unfortunately, the Archimedean copula cannot produce a negative dependence. See Marshall and Olkin (1988) for more detailed discussions about the dependence structure of Archimedean copulas. In the next section, we consider a Gaussian copula and show that a multivariate extension of the Wang transform is derived under some conditions on the aggregate risk $Z$ from Bühlmann's equilibrium pricing formula.

\section{A multivariate EXTENSiOn OF THE WANG TRANSFORM}

In this section, we extend the discussions of Wang (2003) to the multivariate case. For this purpose, we employ a Gaussian copula for the underlying risks $\boldsymbol{X}=\left(X_{1}, X_{2}, \ldots, X_{n}\right)$. That is, define

$$
U_{j} \equiv \Phi^{-1}\left[F_{j}\left(X_{j}\right)\right], \quad j=1,2, \ldots, n,
$$

where $\Phi(x)$ is the standard normal CDF and $F_{j}(x)$ is the marginal CDF for $X_{j}$. It is assumed for the sake of simplicity that $F_{j}(x)$ is strictly increasing in $x$ for all $j$. A Gaussian copula assumes that $\boldsymbol{U}=\left(U_{1}, U_{2}, \ldots, U_{n}\right)$ follows an $n$-variate standard normal distribution with correlation matrix $\boldsymbol{\Sigma}_{\rho}=\left(\rho_{i j}\right)$.

In this copula situation, the joint $\operatorname{CDF} F(\boldsymbol{x})$ for $\boldsymbol{X}$ is given by

$$
F(\boldsymbol{x})=P\left\{X_{1} \leq x_{1}, X_{2} \leq x_{2}, \ldots, X_{n} \leq x_{n}\right\}=P\left\{U_{1} \leq \alpha_{1}, U_{2} \leq \alpha_{2}, \ldots, U_{n} \leq \alpha_{n}\right\},
$$

where $\alpha_{j}=\Phi^{-1}\left[F_{j}\left(x_{j}\right)\right], j=1,2, \ldots, n$. Denote by $\phi_{n}(\boldsymbol{x}), \boldsymbol{x}=\left(x_{1}, x_{2}, \ldots, x_{n}\right)$, the PDF of the $n$-variate standard normal distribution with correlation matrix $\boldsymbol{\Sigma}_{\rho}$, 
$\phi(x)$ the PDF of the univariate standard normal distribution, and $f_{j}(x)$ the marginal PDF of $X_{j}$. Then, the joint $\operatorname{PDF} f(\boldsymbol{x})$ for $\boldsymbol{X}$ exists and is given by

$$
f(\boldsymbol{x})=\phi_{n}(\boldsymbol{\alpha}) \prod_{j=1}^{n} \frac{\mathrm{d} \alpha_{j}}{\mathrm{~d} x_{j}}=\phi_{n}(\boldsymbol{\alpha}) \prod_{j=1}^{n} \frac{f_{j}\left(x_{j}\right)}{\phi\left(\alpha_{j}\right)},
$$

since $\Phi\left(\alpha_{j}\right)=F_{j}\left(x_{j}\right)$.

Now, as in Wang (2003), suppose that the aggregate risk $Z$ consists of many individual risks $X_{j}$ so that it can be approximated by a normal distribution. Let $Z_{0}=\left(Z-\mu_{Z}\right) / \sigma_{Z}$, where $\mu_{Z}=E[Z]$ and $\sigma_{Z}^{2}=V[Z]$, be the standardized normal random variable. Then, from (2.1), we have

$$
\pi(Y)=\frac{E\left[h(\boldsymbol{X}) \mathrm{e}^{-\lambda_{0} Z_{0}}\right]}{E\left[\mathrm{e}^{-\lambda_{0} Z_{0}}\right]}, \quad \lambda_{0}=\lambda \sigma_{Z} .
$$

Next, suppose that the standard normal variable $Z_{0}$ is expressed as

$$
Z_{0} \stackrel{\mathrm{d}}{=} \xi+\sum_{j=1}^{n} w_{j} U_{j}, \quad U_{j}=\Phi^{-1}\left[F_{j}\left(X_{j}\right)\right]
$$

for some constants $w_{j}$ and a random variable $\xi$, which is independent of $U_{j}$, where $\stackrel{d}{=}$ stands for equality in law. It follows from (3.2) that

$$
\pi(Y)=\frac{E\left[h(\boldsymbol{X}) \mathrm{e}^{-\sum_{j=1}^{n} \lambda_{j} U_{j}}\right]}{E\left[\mathrm{e}^{-\sum_{j=1}^{n} \lambda_{j} U_{j}}\right]}, \quad \lambda_{j}=\lambda_{0} w_{j} .
$$

Since $U \equiv \sum_{j=1}^{n} \lambda_{j} U_{j}$ is normally distributed with mean 0 and variance $\lambda^{\top} \boldsymbol{\Sigma}_{\rho} \lambda$ with $\lambda=\left(\lambda_{1}, \lambda_{2}, \ldots, \lambda_{n}\right)$, we obtain

$$
\pi(Y)=\mathrm{e}^{-\sigma_{U}^{2} / 2} E\left[h(\boldsymbol{X}) \mathrm{e}^{-\sum_{j=1}^{n} \lambda_{j} U_{j}}\right], \quad \sigma_{U}^{2} \equiv \lambda^{\top} \boldsymbol{\Sigma}_{\rho} \lambda .
$$

Remark 3.1. The decomposition (3.3) is always possible for normal random variables due to the linearity. However, when considering the joint distribution of $\left(h(\boldsymbol{X}), Z_{0}\right)$, replacing $Z_{0}$ by some $Z^{\prime}$ with $Z \stackrel{\mathrm{d}}{=} Z^{\prime}$ may not result in the same joint distribution. It is therefore of great interest to investigate the accuracy of the approximation (3.3) for the joint distribution of $\left(h(\boldsymbol{X}), Z_{0}\right)$ under the Gaussian copula setting.

Using the approximation (3.3), the change of measure formula (2.4) becomes

$$
f^{*}(\boldsymbol{x})=\mathrm{e}^{-\sigma_{U}^{2} / 2} \mathrm{e}^{-\sum_{j=1}^{n} \lambda_{j} \alpha_{j}} f(\boldsymbol{x}), \quad \alpha_{j}=\Phi^{-1}\left[F_{j}\left(x_{j}\right)\right],
$$


in this setting. It follows from (3.1) that

$$
f^{*}(\boldsymbol{x})=\mathrm{e}^{-\sigma_{U}^{2} / 2} \mathrm{e}^{-\Sigma_{j=1}^{n} \lambda_{j} \alpha_{j}} \phi_{n}(\boldsymbol{\alpha}) \prod_{j=1}^{n} \frac{f_{j}\left(x_{j}\right)}{\phi\left(\alpha_{j}\right)} .
$$

Integrating (3.5) from - $\infty$ to $y_{j}$ for each component, we obtain the CDF under $P^{*}$ as

$$
F^{*}(\boldsymbol{y})=\mathrm{e}^{-\sigma_{U}^{2} / 2} \int^{\boldsymbol{y}} \mathrm{e}^{-\Sigma_{j=1}^{n} \lambda_{j} \alpha_{j}} \phi_{n}(\boldsymbol{\alpha}) \prod_{j=1}^{n} \frac{f_{j}\left(x_{j}\right)}{\phi\left(\alpha_{j}\right)} \mathrm{d} \boldsymbol{x}, \quad \boldsymbol{y}=\left(y_{1}, y_{2}, \ldots, y_{n}\right) .
$$

We now employ the change of variables; $u_{j}=\Phi^{-1}\left[F_{j}\left(x_{j}\right)\right]$. Then, since $\alpha_{j}=u_{j}$, we obtain

$$
F^{*}(\boldsymbol{y})=\mathrm{e}^{-\sigma_{U}^{2} / 2} \int^{\boldsymbol{\beta}} \mathrm{e}^{-\Sigma_{j=1}^{n} \lambda_{j} u_{j}} \boldsymbol{\phi}_{n}(\boldsymbol{u}) \mathrm{d} \boldsymbol{u}, \quad \beta_{j}=\Phi^{-1}\left[F_{j}\left(y_{j}\right)\right],
$$

with $\boldsymbol{\beta}=\left(\beta_{1}, \beta_{2}, \ldots, \beta_{n}\right)$. Note that, from (2.10), we have

$$
\mathrm{e}^{-\sigma_{U}^{2} / 2} \mathrm{e}^{-\Sigma_{j=1}^{n} \lambda_{j} u_{j}} \phi_{n}(\boldsymbol{u})=\phi_{n}\left(\boldsymbol{u}+\boldsymbol{\Sigma}_{\rho} \lambda\right) .
$$

Therefore, we finally obtain from (3.6) that

$$
F^{*}(\boldsymbol{y})=\Phi_{n}\left(\boldsymbol{\beta}+\boldsymbol{\Sigma}_{\rho} \lambda\right) .
$$

More precisely, we obtain a multivariate extension of the Wang transform as follows:

$$
F^{*}(\boldsymbol{x})=\Phi_{n}\left(\Phi^{-1}\left[F_{1}\left(x_{1}\right)\right]+\sum_{j=1}^{n} \lambda_{j} \rho_{1 j}, \ldots, \Phi^{-1}\left[F_{n}\left(x_{n}\right)\right]+\sum_{j=1}^{n} \lambda_{j} \rho_{n j}\right) .
$$

Note that, when $n=1$, (3.7) coincides with the Wang transform (1.2) since $\rho_{11}=1$.

Remark 3.2. Since actual market data exhibit the fat-tailed character, it is wise to replace the normal distribution with a fat-tailed distribution such as $t$-distributions, as suggested in Wang (2002). However, according to the above derivation, the $n$-variate standard normal distribution $\Phi_{n}$ used in (3.7) comes from the Gaussian copula to specify the dependence structure of the underlying risks. Because Bühlmann's equilibrium pricing formula does not apply for the case that the underlying risks follow a multivariate $t$-distribution, it is not possible to use $t$-copulas directly for $\left(U_{1}, U_{2}, \ldots, U_{n}\right)$ in (3.3). A further investigation is required to develop such pricing models in this framework. See Kijima and Muromachi (2006) for some development in this direction. 


\subsection{The case of normal distributions}

When the underlying risks follow an $n$-variate normal distribution, equation (3.3) holds true, not just an approximation. Hence, in this special case, the multivariate Wang transform (3.7) coincides with the multivariate Esscher transform (2.4), as we shall see below.

Suppose that $\boldsymbol{X}=\left(X_{1}, X_{2}, \ldots, X_{n}\right)$ has mean vector $\boldsymbol{\mu}=\left(\mu_{1}, \mu_{2}, \ldots, \mu_{n}\right)$ and covariance matrix $\Sigma=\left(\sigma_{i j}\right)$. Then, $U_{j}=\left(X_{j}-\mu_{j}\right) / \sigma_{j}$, where $\sigma_{j j}=\sigma_{j}^{2}$, in this special case. Since $Z=\sum_{j=1}^{n} X_{j}$, we have

$$
Z_{0}=\frac{Z-\mu_{Z}}{\sigma_{Z}}=\sum_{j=1}^{n} \frac{X_{j}-\mu_{j}}{\sigma_{Z}} .
$$

Hence, taking $w_{j}=\sigma_{j} / \sigma_{Z}$ and $\xi=0$, we obtain (3.3) with the ordinary equality, not equality in law.

By the definition of $\lambda_{j}$, we have

$$
\lambda_{j}=\lambda \sigma_{Z} \frac{\sigma_{j}}{\sigma_{Z}}=\lambda \sigma_{j}, \quad j=1,2, \ldots, n,
$$

so that

$$
\sum_{j=1}^{n} \lambda_{j} \rho_{i j}=\lambda \operatorname{Cov}\left(U_{i}, Z\right), \quad i=1,2, \ldots, n .
$$

Hence, in the normal case, the Wang transform (3.7) is given by

$$
F^{*}(\boldsymbol{x})=\Phi_{n}\left(\Phi^{-1}\left[F_{1}\left(x_{1}\right)\right]+\frac{\lambda}{\sigma_{1}} \operatorname{Cov}\left(X_{1}, Z\right), \ldots, \Phi^{-1}\left[F_{n}\left(x_{n}\right)\right]+\frac{\lambda}{\sigma_{n}} \operatorname{Cov}\left(X_{n}, Z\right)\right) .
$$

Since $F_{j}\left(x_{j}\right)=\Phi\left(\left(x_{j}-\mu_{j}\right) / \sigma_{j}\right)$ in this case, we conclude that the multivariate Wang transform (3.8) distorts the normal distribution to another normal distribution with mean vector $\boldsymbol{\mu}-\lambda \boldsymbol{\Sigma} \mathbf{1}$ and the same covariance matrix $\boldsymbol{\Sigma}$, which coincides with the multivariate Esscher transform; see Theorem 2.2.

\subsection{The case of log-normal distributions}

When the underlying risks $\boldsymbol{X}=\left(X_{1}, X_{2}, \ldots, X_{n}\right)$ follow a multivariate log-normal distribution, the Esscher transform cannot define the pricing formula, while does the Wang transform (3.7). This is one of the advantages of the Wang transform over the Esscher counterpart.

Suppose that $\left(\log X_{1}, \log X_{2}, \ldots, \log X_{n}\right)$ has mean vector $\boldsymbol{\mu}=\left(\mu_{1}, \mu_{2}, \ldots, \mu_{n}\right)$ and covariance matrix $\boldsymbol{\Sigma}=\left(\sigma_{i j}\right)$. In this case, contrary to the normal case, there is no reason to specify $w_{j}=\sigma_{j} / \sigma_{Z}$ and $\xi=0$. However, if we take the choice, we then obtain the transform (3.8), with $X_{i}$ being replaced by $\log X_{i}$, even for this case. Since $F_{j}\left(x_{j}\right)=\Phi\left(\left(\log x_{j}-\mu_{j}\right) / \sigma_{j}\right)$ for $\log$-normal distributions, we conclude that the multivariate Wang transform (3.8) distorts the log-normal distribution 
to another log-normal distribution with mean vector $\boldsymbol{\mu}-\lambda \boldsymbol{\Sigma} \mathbf{1}$ and the same covariance matrix $\boldsymbol{\Sigma}$.

\section{ACKNOWLEDGMENTS}

The author acknowledges fruitful conversations with Dr. Shaun Wang and and Dr. Yukio Muromachi. He is also grateful to anonymous referees for their invaluable comments that improved the original manuscript considerably.

\section{REFERENCES}

Black, F. and Scholes, M. (1973) The pricing of options and corporate liabilities, Journal of Political Economy, 81, 637-654.

Bühlmann, H. (1980) An economic premium principle, Astin Bulletin, 11, 52-60.

Gerber, H.U. and SHiu, E.S.W. (1994) Option pricing by Esscher transforms, Transactions of the Society of Actuaries, 46, 99-140.

HARrison, M.J. and KREPS, D. (1979) Martingales and arbitrage in multiperiod securities market, Journal of Economic Theory, 20, 381-408.

Iwaki, H., KiJima, M. and MoRimoto, Y. (2001) An economic premium principle in a multiperiod economy, Insurance: Mathematics and Economics, 28, 325-339.

Jensen, L.J. (1995) Saddlepoint Approximation, Oxford Science Publications, Oxford.

Kisima, M. (2002) Stochastic Processes with Applications to Finance, Chapman \& Hall, London.

Kisima, M. and Muromachi, Y. (2001) Pricing of equity swaps in a stochastic interest rate economy. Journal of Derivatives, 8, 19-35.

Kisima, M. and Muromachi, Y. (2006) On the Wang transform with fat-tail distributions. In Preparation.

KiJima, M. and OHNishi, M. (1996) Portfolio selection problems via the bivariate characterization of stochastic dominance relations, Mathematical Finance, 6, 237-277.

Marshall, A.W. and Olkin, I. (1988) Families of multivariate distributions. Journal of the American Statistical Association, 83, 834-841.

Nelsen, R.B. (1999) An Introduction to Copulas, Springer, New York.

PLISKA, S.R. (1997) Introduction to Mathematical Finance: Discrete Time Models, Blackwell, Cambridge.

Tong, Y.L. (1990) The Multivariate Normal Distribution, Springer, New York.

WANG, S.S. (2000) A class of distortion operators for pricing financial and insurance risks, Journal of Risk and Insurance, 67, 15-36.

WANG, S.S. (2002) A universal framework for pricing financial and insurance risks, Astin Bulletin, 32, 213-234.

WANG, S.S. (2003) Equilibrium pricing transforms: New results using Bühlmann's 1980 economic model, Astin Bulletin, 33, 57-73.

WANG, S.S. (2005) Normalized exponential tilting: Pricing and measuring multivariate risks, Working Paper, Georgia State University.

MASAAKI KiJIMA

Daiwa Securities Chair,

Graduate School of Economics,

Kyoto University, Yoshida-Honmachi, Sakyo-ku,

Kyoto 606-8501, Japan.

TellFax: +81-75-753-3511

E-Mail:kijima_daiwa@econ.kyoto-u.ac.jp 